\title{
Wastewater reuse applications and contaminants of emerging concern
}

\author{
Despo Fatta-Kassinos $\cdot$ Costas Michael
}

Received: 13 March 2013 / Accepted: 28 March 2013 / Published online: 24 April 2013

(C) Springer-Verlag Berlin Heidelberg 2013

In response to the escalating problem of water shortage all over the world, treated wastewater is nowadays widely reused and is generally considered as a reliable alternative water source. Although the reuse practice is accompanied by a number of benefits relating to the enhancement of water balances and soil nutrition by the elements existing in the treated flows, a number of unanswered questions still puzzle scientists. Further to the lack of unequivocal knowledge on the possible elemental interactions that can affect the accumulation of heavy metals and other elements in the soil and their uptake by biota, during the last 2 decades, a number of organic contaminants of emerging concern has been identified and quantified in environmental compartments. The latter was made possible due to the remarkable technological progress in relation to analytical chromatographic methods, which enabled the identification and quantification of such compounds down to the $\mathrm{ng} / \mathrm{L}$ level. Moreover, it is now known that the effluents' remaining organic matter after conventional treatment consists of a number of recalcitrant organic xenobiotic compounds including potential endocrine disrupting compounds, antibiotics, and others. The fact that the currently applied treatment processes for urban wastewater abatement fail to remove completely such contaminants leading to their subsequent release in the terrestrial and aquatic environment through disposal and reuse applications is widely accepted. The number of studies focusing on the analysis and the toxicological assessment of such compounds in the environment is constantly increasing the aim being to bridge the various knowledge gaps associated with these issues.

Responsible editor: Philippe Garrigues

D. Fatta-Kassinos $(\bowtie) \cdot$ C. Michael

Nireas-International Water Research Center, University of Cyprus, P.O. Box 20537, 1678 Nicosia, Cyprus

e-mail: dfatta@ucy.ac.cy

C. Michael

e-mail: tsimicha@spidernet.com.cy
On the 13th and 14th of September 2012, approximately 80 scientists gathered in Limassol, Cyprus, to discuss the challenges of wastewater reuse practices with regard to contaminants of emerging concern, their transformation while in the environment, their potential uptake by plants and crops, the effects that these contaminants may induce in the environment, the evolution and release of antibiotic resistance, and the technologies that are able to remove such contaminants from wastewater. The workshop addressed the following themes:

- Which are the contaminants of emerging concern that are relevant to wastewater reuse applications? (e.g., antibiotics and other licit and illicit drugs, transformation products, disinfection byproducts)

- What are the new concerns related to reuse applications? (direct and/or indirect effects)

- What technologies can enhance the "conventional" treatment by removing such contaminants?

The workshop was held jointly by the network of reference laboratories for monitoring of emerging environmental pollutants (NORMAN), COST Action TD0803 detecting evolutionary hot spots of antibiotic resistances in Europe (DARE) and the Nireas-International Water Research Center. NORMAN (www.norman-network.net) is an independent and competent platform in the field of emerging pollutants. NORMAN facilitates exchange of information, debate, and research collaboration at a global level with official recognition from institutional agencies of Europe. The main objective of DARE, EU COST Action TD0803 (www.cost-dare.eu), is to identify and characterize environmental hotspots for the emergence of antimicrobial resistance and the spread of antibiotics and antibiotic resistance patterns, aiming to develop measures to control the evolution of antibiotic resistance. Great emphasis is given to Wastewater Treatment Plants, which constitute a major source for the release of (newly evolved) antibiotic resistances into the environment. 
Nireas-International Water Research Center is co-funded by the European Regional Development Fund and the Republic of Cyprus through the Cyprus Research Promotion Foundation (NEA $\Upsilon \Pi \mathrm{O} \Delta \mathrm{OMH} / \Sigma \mathrm{TPATH} / 0308 / 09$ ). It was created with the objective of leveraging scientific and engineering expertise in order to tackle water-related problems. (http://www.nireas-iwrc.org).

Highly interesting and scientifically stimulating observations were postulated during the event, which can be summarized in the following:

- Information on the effects of the inadvertent chronic exposure of organisms to micropollutants is scarce and incomplete.

- Long-term effects of micropollutants, including those at the sublethal level need to be assessed and understood.

- The tests and methods for assessing the adverse effects of micropollutants in wastewater are not standardized (i.e., validated protocols are required). Reuse applications should integrate phytoxicity/ecotoxicity tests.

- The uptake of micropollutants by plants and crops during wastewater reuse applications has been proved in many cases. More systematic studies under real environmental conditions are required.

- Effluent quality criteria are required in order to assess the various technologies in a uniform way during reuse practices.

- Treatment technologies need to be evaluated on a uniform basis in respect to their capacity to remove micropollutants, antibiotic resistance, produce unwanted transformation products and remove/induce toxicity, etc. The operation cost of such technologies needs to be determined so that comparison of technologies could be feasible, taking into account their efficiency in removing the aforementioned compounds.

- Antibiotic resistance genes should be considered as contaminants of emerging concern. Information on the capacity of the currently applied wastewater treatment systems in removing antibiotic resistance is still scarce.

- Concerning antibiotic resistance, model sites in various countries should be examined in relation to wastewater reuse implemented schemes.

- The tests and methods for assessing antibiotic resistance removal are not standardized (uniform protocols are required).

Eleven papers were accepted and included in this special issue. These papers discuss the new trends in the analytical determination of contaminants of emerging concern and their transformation products in environmental waters, and also whether the evaluation of "traditional" physicochemical parameters in wastewater is sufficient to explain the potential toxicity of the treated wastewater at the sewage treatment plants. They also elaborate on the impact of treated wastewater irrigation on antibiotic resistance in the soil microbiome, the subinhibitory concentrations of antibiotics and wastewater influencing biofilm formation and gene expression of multiresistant Pseudomonas aeruginosa, and highlight current research in human health implications of clinically relevant bacteria in wastewater. They present a comparison of photocatalytic degradation of dyes in wastewater in relation to their structure, the photocatalytic degradation of contaminants with composite $\mathrm{NF}^{-\mathrm{TiO}_{2}}$ films under visible and solar light, the fate of $\mathrm{x}$-ray contrast media during microbial degradation in an MBBR system, the removal of highly polar compounds from wastewater by powdered activated carbon, the removal of contaminants of emerging concern by adsorption, and finally, the uptake of microcontaminants by crops irrigated with reclaimed water and groundwater under real field conditions.

The great interest of the scientists who participated in the event on wastewater reuse led to the submission of a proposal for the establishment of a working group (WG) to be operated under the NORMAN network. The Steering Committee of the network has approved in January 2013 the establishment of the new task force based on the discussions undertaken during its general assembly meeting in November 2012. The WG will be led by Nireas-International Water Research Center. The WG will be steered by scientists participating in the COST Action TD0803 DARE, the NORMAN network, and also scientists and researchers active in the particular field. It is expected that the WG will establish a new strong scientific and technological platform that will be able to progress the relevant state-of-theart and advance further current understanding.

The guest editors would like to thank Dr. Valeria Dulio and Dr. Jaroslav Slobodnik (Executive Secretary and Chairman of NORMAN Network), and Professor Thomas Berendonk (Chair of COST Action TD0803) for the organization of the workshop and for sharing new ideas that hold promising scientific potential. The guest editors would also like to thank all authors contributing to this issue, and also, the many reviewers called upon to offer constructive advice for the improvement of submissions. They extend their sincere thanks to Springer for the opportunity provided to compile such a special issue, and would like to thank in particular the Editor-in-Chief of ESPR, Dr. Philippe Garrigues and Editorial Assistant of the journal, Ms. Emmanuelle Pignard-Péguet, for their great support and patience at every stage in the preparation of the issue. 


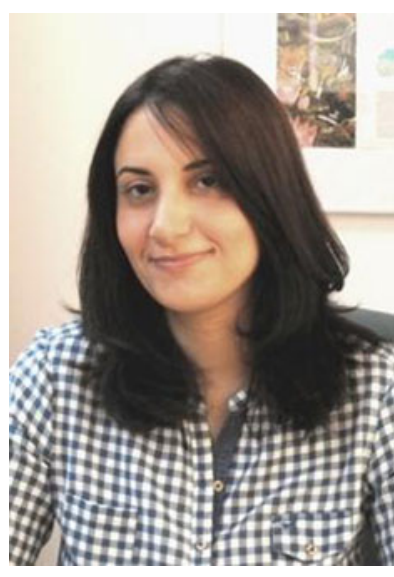

Dr. Despo Fatta-Kassinos received a PhD from the School of Chemical Engineering of the National Technical University of Athens, Greece, where she has also carried postdoctoral research. She is currently an Assistant Professor in the Department of Civil and Environmental Engineering, and Director of Nireas-International Water Research Center, at the University of Cyprus. Her research centers on the understanding of the fate and behavior of emerging contaminants during advanced water

and wastewater treatment. In specific, the activities of her research group include the identification of xenobiotic compounds in aqueous samples, the assessment of their environmental impact, the development of new technological processes for their degradation and removal from aqueous media, and wastewater reuse applications. She has over 80 peer-reviewed scientific publications in SCI journals, several book chapters and co-edited the book 'Xenobiotics in the urban water cycle' published by Springer. She is the recipient of 'Nikos Symeonides 2011' national research prize, awarded by the Cyprus Research Promotion Foundation in 2012. She serves as editor for the Journal of Environmental Chemical Engineering, Elsevier and she is an elected member of the Scientific and Technological Board of Joint Programming Initiative 'Water Challenges for a Changing World'.

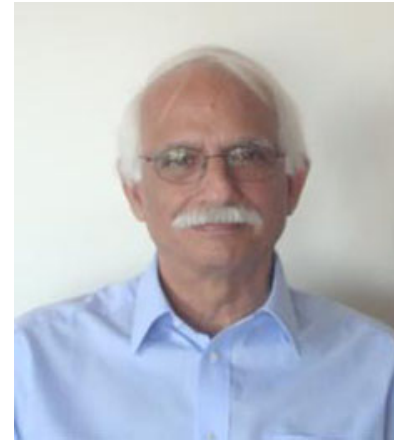

Dr Costas Michael holds a $\mathrm{PhD}$ degree in Chemistry from Athens University, Greece. He has been Lecturer and then Assistant Professor at the Department of Biological Chemistry, School of Medicine, Athens University. He worked for almost 30 years at the multidisciplinary State General Laboratory in Cyprus from which he left as its Director. He has long experience in the separation, identification and quantitation of organics and inorganics at trace levels, particularly in the domain of the environment, with state-of-the-art instrumentation. He is now a senior scientist at GAIA-Laboratory of Environmental Engineering and a member of the Board of Directors of the Nireas-International Water Research Center at the University of Cyprus, where he also serves as a special teaching scientist both at the undergraduate and postgraduate programmes. He is a member of the Editorial Board of the ESPR. His research interests are in the environmental measurement and assessment with focus on wastewater quality and treatment and the removal of xenobiotics from waters and wastewaters through advanced oxidation processes. He has published over 45 papers in peer review journals and in the proceedings of national and international refereed conferences. He has participated in many National and EU funded projects. 